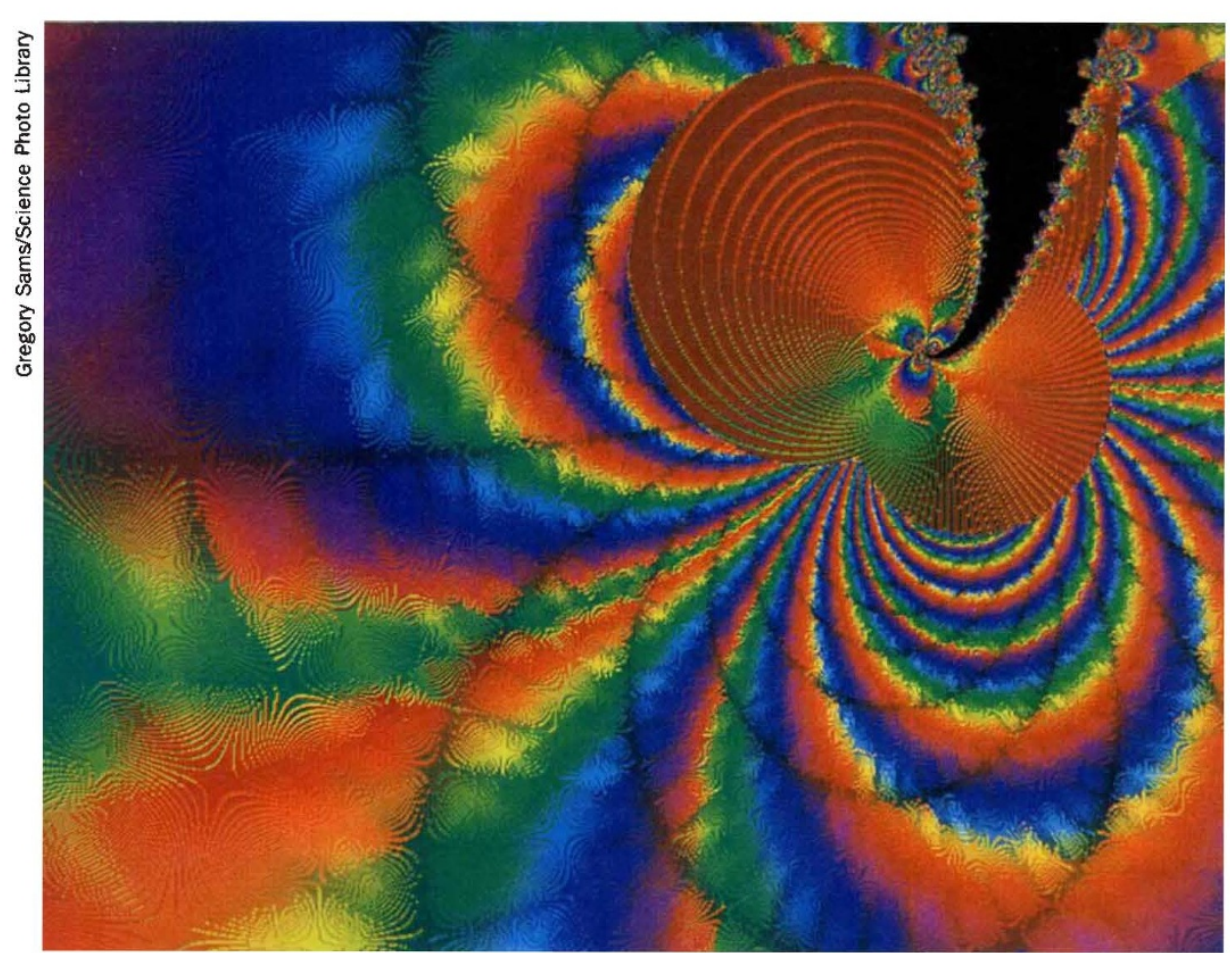

The art of the impossible: a computer-generated picture of the uncomputable Julia set.

by checking all possible sums (infinitely many) of two $n$th powers. More ambitiously, it could prove all possible theorems in a finite period of time, by pursuing all logically valid chains of deduction from the axioms of set theory. It is clear from this that some 'classical' dynamical systems can simulate bizarre computations. The reason is that perfectly respectable equations have a natural interpretation as models of physically impossible devices.

However, it is far from trivial to implement this informal project in a logically impeccable manner. For example, one must actually write down the equations - or at least indicate how this could be done. And one must deal with the potentially infinite-dimensional space of positions on the Turing machine's tape. da Costa and Doria use a quite different approach. Their basic idea is inspired by a remark of B. Scarpellini ${ }^{8}$ that it may be possible "to build an analog computer that can simulate functions $f(x)$ so that the predicate $[\phi(f(x))]$ isn't decidable, while the analog machine itself decides it". Developing this idea, da Costa and Doria construct a series of functions $f_{m}(x)$, obtained from classical functions and integrals that involve them. These functions are either identically 0 or identically 1 , but their value is formally undecidable.

da Costa and Doria define a family of classical (that is, not quantum) dynamical systems, one for each $m$, each being either a free particle or a simple harmonic oscillator, depending upon the value of $f_{m}$. Since the value of $f_{m}$ is undecidable, there is no formal way to compute which is the case. However, one can imagine a thought experiment in which an analog device simulates the dynamics for any given $m$. It will then be apparent to an observer whether or not the motion is simple harmonic or free (this choice is Scarpellini's predicate $\phi$ ) - in one case the particle oscillates along an interval, in the other it whizzes off to infinity - so the analog machine can effectively decide the undecidable. In this thought experiment, a classical system computes something that is algorithmically undecidable, contrary to Penrose's thesis.

The thrust of Penrose's thesis is thus directed elsewhere: if it is to be valid, then it must be posed for real classical systems rather than mathematical ones, a concept that is hard to handle rigorously in a world that is really quantum. Clearly some rethinking is in order here. Dynamical systems theorists may object that the da Costa-Doria family is artificial and thus has no implications for systems that actually arise in applications. It is worth remembering that similar objections were raised to the work of Turing and Gödel.

Ian Stewart is in the Mathematics Institute, University of Warwick, Coventry CV4 7AL, UK.

1. Penrose, R. The Emperor's New Mind (Oxford University
Press, 1990)

2. da Costa, N. C. A. Foundations Phys. (in the press)

3. Doria, F. A. Int. J. theor. Phys. (in the press).

4. Benedicks, M. \& Carleson, L. Ann. Math. 133, 73-169 (1991).

6. Bennett, C. H. Nature 346, 606-607 (1990)

7. Blum, L., Shub M. \& Smale, S. Bull. Am. math. Soc. 21 1- 46 (1989).

8. Scarpellini, B. Z. math. Logik 9, 265 (1963)
5. Moore, C. Phys. Rev. Lett. 64, 2354-2357 (1990).

\section{In the gap}

DeLetions of a portion of the long arm of chromosome 15 are associated with two phenotypically distinct disorders,

Prader-Willi syndrome and Angelman syndrome; the difference depends on which parental chromosome suffers the deletion, and provides strong evidence for genetic imprinting. J. Wagstaff et al (Am. Soc. hum. Genet. 49, 330-337; 1991) now report the first known gene to map to this region (15q11-13), that encoding the $\beta 3$ subunit of the GABA ( $\gamma$-aminobutyric acid) receptor. Although one copy of the gene is commonly deleted in people suffering from the syndromes, both copies were present in one Prader-Willi patient with an unbalanced chromosomal translocation Nevertheless, the possible involvement of this neurotransmitter receptor in the pathogenesis of the two disorders will no doubt now be the subject of detailed scrutiny.

\section{Binges and bagels}

PEOPLE like to eat, and they like it more when they do it with other people. The more the merrier, says J. M. De Castro in Physiology \& Behaviour (49, 1289 1291 ; 1991), who paid 315 people $\$ 30$ each to keep 7-day diaries recording everything they ate, where and when they ate it, and how many other people kept them company. The correlation between meal size and company remained rock-solid even when weekend binges and drunken excesses on a Saturday night were taken into account. Social facilitation, claims De Castro, could be the most important determinant of normal human eating behaviour. The results corroborate the old story of the rabbi at a social function who was offered another bagel. "No, I couldn't possibly" he says, "I've already had six". "You've had seven", came the reply, "but who's counting?".

\section{Seeing red}

G. Albrecht-Buehler ( . Cell Biol. 114 493-502; 1991), asking whether cultured cells will respond to infrared light, has received an affirmative answer. When tickled with a few quanta, 3T 3 cells it seems (some of them at least) extend pseudopodia towards the light source. The preferred wavelength is $800-900 \mathrm{~nm}$ and pulsed irradiation generates the most activity. The heating effect is negligible so the cells must have a chromophoric receptor, a pigment presumably (though it ought to have absorption bands at shorter wavelengths also). And how does this curious faculty profit the cells? AlbrechtBuehler allows himself some fairly uninhibited speculations: perhaps infrared intensity is attenuated by a chemotactic concentration gradient, which the cells might then more easily sense, or perhaps the cell is an emitter as well as detector of radiation and exchanges signals with its friends. But could the process simply help them to avoid bumping into things in the dark? 